\title{
Hépatotoxicité à la suite de l'utilisation à court terme d'amiodarone pour une personne âgée : un rapport de cas
}

\author{
par Jessica Ferguson, Anne-Isabelle Dubé et Catherine Ouellet
}

\section{INTRODUCTION}

$\mathrm{L}$ 'amiodarone, un anti-arythmique de classe III, est utilisée pour traiter une vaste gamme d'arythmies auriculaires et ventriculaires. Bien que l'amiodarone soit cliniquement efficace, l'utilisation de cet agent est limitée, tant pour la population âgée que pour les plus jeunes patients, à cause de sa forte toxicité, particulièrement sur le plan cardiaque, pulmonaire et thyroïdien. La toxicité hépatique de l'amiodarone reste un effet indésirable peu connu et peu rapporté dans la littérature.

\section{PRÉSENTATION DU CAS}

Une patiente de 75 ans est admise à une unité de médecine interne d'un hôpital universitaire en raison de la présence de méléna associée à une anémie importante (jour 0 [J0]). Le diagnostic permet d'établir que ces symptômes sont attribuables à une hémorragie digestive secondaire à la présence de télangiectasies intestinales. La patiente est déjà connue pour différents problèmes de santé et des allergies, qui sont résumés à la boîte 1. L'épisode d'hémorragie digestive est pris en charge, mais l'hospitalisation de la patiente est prolongée en raison de multiples complications, dont le développement d'une arthrite septique à Staphylococcus aureus (sensible à la méthicilline) à l'épaule gauche ainsi que deux épisodes d'infarctus du myocarde sans élévation du segment ST aux jours 11 et 21 de l'hospitalisation. De plus, le $18^{\mathrm{e}}$ jour après son admission $(\mathrm{J}+18)$, la patiente présente un épisode de fibrillation auriculaire soutenu. $\mathrm{Au} \mathrm{J}+22$, on entreprend l'administration de métoprolol à raison de $50 \mathrm{mg}$ per os deux fois par jour afin d'obtenir un contrôle de la réponse ventriculaire. Puis, à la suite d'une proposition émanant du département de cardiologie, qui souhaite instaurer un traitement de maintien, l'amiodarone lui est prescrit au J+26 à une dose de $600 \mathrm{mg}$ per os deux fois par
Boîte 1. Antécédents médicaux et allergies de la patiente lors de l'hospitalisation (jour 0 )

Antécédents médicaux

Sclérodermie associée au syndrome de CREST, depuis 1995

(sclérodermie limitée au syndrome de Raynaud et à la

présence de télangiectasies intestinales)

Syndrome d'ectasies vasculaires antrales

Insuffisance veineuse des membres inférieurs

Sténose spinale multiétagée de L3 à S1

Gonarthrose bilatérale

Syndrome des jambes sans repos

Pseudogoutte

Syndrome d'apnée et d'hypopnée du sommeil

Hypothyroïdie

Hypertension

Dyslipidémie

Trouble dépressif

Allergie/intolérance

Nifédipine : type de réaction inconnu

Pénicilline : rash

jour en ajout au bêta-bloqueur. Au J+35, voyant que le bilan hépatique de la patiente montre une augmentation marquée des transaminases, l'équipe traitante réduit la dose d'amiodarone à $200 \mathrm{mg}$ per os deux fois par jour. La liste complète de la médication active en date du J+35 est inscrite au tableau 1 . Malgré cette réduction de dosage, les enzymes hépatiques poursuivent leur augmentation progressive et, dans ce contexte, l'équipe médicale cesse les traitements qu'elle considère comme ayant des effets potentiellement indésirables sur le plan hépatique. L'amiodarone et la pravastatine, deux traitements qui ont été entrepris durant l'hospitalisation, sont donc complètement interrompus au J+39, et seul le métoprolol est conservé dans le cadre du traitement de la fibrillation 
This single copy is for your personal, non-commercial use only.

For permission to reprint multiple copies or to order presentation-ready copies for distribution, contact CJHP at cjhpedit@cshp.ca

\section{Tableau 1. Médication active au dossier en date du jour +35 après l'hospitalisation}

\begin{tabular}{ll} 
Médication & \multicolumn{1}{c}{ Dose } \\
\hline Cloxacilline & $2 \mathrm{~g}$ intraveineux toutes les 6 heures \\
Amiodarone & $600 \mathrm{mg}$ per os 2 fois par jour \\
Métoprolol & $50 \mathrm{mg}$ per os 2 fois par jour \\
Furosémide & $20 \mathrm{mg}$ per os 1 fois par jour \\
Chlorure de potassium & $20 \mathrm{mmol}(20 \mathrm{mÉq})$ per os 3 fois par jour \\
Acide acétylsalicylique & $80 \mathrm{mg}$ per os 1 fois par jour \\
Pravastatin & $40 \mathrm{mg}$ per os 1 fois par jour \\
Nitroglycérine, timbre de 0,8 mg/h & 1 timbre $12 \mathrm{~h} / 24 \mathrm{~h}$ \\
Venlafaxine XR & $75 \mathrm{mg}$ per os 1 fois par jour \\
Lévothyroxine & $0,075 \mathrm{mg}$ per os 1 fois par jour \\
Éthinylestradiol 0,03 mg et noréthindrone & $1 \mathrm{comprimé}$ per os 1 fois par jour \\
1,5 mg (LoEstrin) & \\
Ésomeprazole & $40 \mathrm{mg}$ per os 1 fois par jour \\
Métoclopramide & $10 \mathrm{mg}$ per os 3 fois par jour, au besoin \\
Carbonate de calcium & $500 \mathrm{mg}$ per os 1 fois par jour \\
Cholécalciferol & 10 000 Ul per os 1 fois par semaine \\
Héparine & 5000 unités sous-cutanées 2 fois par jour \\
Hydromorphone & $0,5 \mathrm{mg}$ per os 4 fois par jour, au besoin \\
Docusate sodique & $200 \mathrm{mg}$ per os 1 fois par jour \\
\hline
\end{tabular}

auriculaire. Dès le J+40, les transaminases hépatiques commencent à diminuer, et une normalisation s'effectue progressivement sur une période de plusieurs jours. L'évolution détaillée des concentrations d'enzymes hépatiques est présentée au tableau 2.

\section{DISCUSSION}

D'un point de vue pharmacocinétique, l'amiodarone est bien connue pour son très grand volume de distribution estimé à 500 litres, avec une cinétique de distribution multicompartimentale $^{1,2}$. Une fois distribué, le médicament se retrouve dans le sang ainsi que dans de nombreux organes et tissus graisseux, principalement les poumons et le foie. Plusieurs semaines, voire même plusieurs mois, peuvent être nécessaires pour atteindre un équilibre entre les concentrations tissulaires et plasmatiques. Ces paramètres de distribution conferent à l'amiodarone une très longue demi-vie d'environ 60 jours. Avant d'être éliminé, ce médicament subit un métabolisme hépatique, principalement par le cytochrome P450 3A4, pour produire la deséthylamiodarone (DEA), son métabolite actif. On ne dispose pas, actuellement, de données sur l'activité de la DEA chez l'homme, mais des études animales ont démontré qu'elle possède des propriétés électrophysiologiques et antiarythmiques notables. Par contre, du point de vue de l'innocuité, l'amiodarone est aussi bien connue pour les nombreux effets secondaires auxquels elle est associée. Outre ses effets indésirables cardiaques, l'amiodarone cause une panoplie d'effets extracardiaques, dont des troubles hépatiques.

Les propriétés pharmacocinétiques de l'amiodarone sont propices à l'apparition d'une hépatotoxicité après un usage à long terme du médicament. Cet effet indésirable peut être lié à la dose cumulative, quoique certains auteurs soutiennent que les concentrations sériques d'amiodarone à l'équilibre peuvent également être en cause ${ }^{3,4}$. L'administration d'amiodarone peut aussi occasionner une élévation rapide et transitoire de l'aspartate aminotransférase (AST) et de l'alanine aminotransférase (ALT) après une courte durée d'utilisation. Cela se produit dans 3 à $55 \%$ des cas selon les références consultées ${ }^{3-7}$. Les concentrations sériques des transaminases peuvent effectivement doubler sans être accompagnées de perturbations de la phosphatase alcaline, de la bilirubine, ni de manifestations cliniques de dysfonction hépatique. Des observations ont démontré que ce type de manifestation se normalise après une diminution de la dose ou la cessation du médicament ${ }^{8}$. Une méta-analyse regroupant des données recueillies auprès de plus de 1400 sujets conclut que l'administration d'amiodarone à faible dose ( $\leq 400 \mathrm{mg}$ par jour) ne comporte pas plus de risque d'hépatotoxicité qu'un placebo après un an de traitement ${ }^{9}$. Bien que ces données semblent rassurantes, il faut considérer qu'en général les patients utilisent l'amiodarone, durant de plus longues périodes. Le fait d'avoir uniquement des données sur une année de traitement constitue donc une limite de cette méta-analyse. Des atteintes hépatiques symptomatiques plus graves ont également été observées dans $3 \%$ des cas, principalement sous forme d'hépatite pouvant même progresser jusqu’à la cirrhose ${ }^{3,5}$. Ce type de réaction peut résulter d'une atteinte hépatique directe ou encore d'une réaction métabolique idiosyncrasique ${ }^{3,8}$. On observe que les rapports de cas et les autres données disponibles dans la littérature médicale témoignent d'une certaine hétérogénéité par rapport aux effets hépatiques que peut entrainer l'amiodarone. 
This single copy is for your personal, non-commercial use only.

For permission to reprint multiple copies or to order presentation-ready copies for distribution, contact CJHP at cjhpedit@cshp.ca

\section{Tableau 2. Évolution des enzymes hépatiques}

\begin{tabular}{|c|c|c|c|c|c|}
\hline Jour & AST (U/L) & ALT (U/L) & $\begin{array}{l}\text { Phosphatase } \\
\text { alcaline (U/L) }\end{array}$ & GGT (U/L) & $\begin{array}{c}\text { Bilirubine } \\
\text { totale }(\mu \mathrm{mol} / \mathrm{L})\end{array}$ \\
\hline 1 & 16 & 11 & 52 & 11 & 9,6 \\
\hline 10 & 30 & 22 & 102 & 51 & 19,4 \\
\hline 11 & 46 & 38 & 118 & - & 17,4 \\
\hline 21 & - & - & - & - & 27,5 \\
\hline 22 & 48 & 86 & 115 & 44 & 22,4 \\
\hline 26 & \multicolumn{5}{|c|}{ Ajout de l'amiodarone à 600 mg per os 2 fois par jour } \\
\hline 35 & 326 & 135 & 208 & - & 19,8 \\
\hline \multicolumn{6}{|c|}{ Diminution de la dose d'amiodarone à 200 mg per os 2 fois par jour } \\
\hline 36 & 346 & 144 & 218 & - & 19,9 \\
\hline 37 & 453 & 180 & 264 & - & 12,2 \\
\hline 38 & 437 & 188 & 264 & - & 16,7 \\
\hline 39 & 472 & 210 & 336 & - & 19,1 \\
\hline \multicolumn{6}{|c|}{ Arrêt de l'amiodarone } \\
\hline 40 & 455 & 216 & 360 & - & 15,7 \\
\hline 42 & 406 & 188 & 387 & 172 & 19,3 \\
\hline 44 & 376 & 166 & 353 & - & 12,5 \\
\hline 46 & 233 & 125 & 281 & 118 & 9,7 \\
\hline 47 & 231 & 121 & 261 & 113 & 13,1 \\
\hline 48 & 237 & 116 & 243 & 107 & 9,5 \\
\hline 50 & 183 & 97 & 201 & 92 & 13,5 \\
\hline 51 & 154 & 86 & 179 & 82 & 12,1 \\
\hline 53 & 119 & 72 & 173 & - & 12,4 \\
\hline 60 & 147 & 69 & 164 & - & 14,8 \\
\hline 61 & 192 & 79 & - & - & - \\
\hline 62 & 193 & 84 & - & - & 14,0 \\
\hline 65 & 110 & 66 & 171 & - & 17,6 \\
\hline 67 & 84 & 51 & 155 & - & 20,7 \\
\hline 75 & 70 & 41 & 155 & - & 25,6 \\
\hline 80 & 74 & 43 & 162 & - & 35,9 \\
\hline 82 & 52 & 36 & 152 & - & 23,8 \\
\hline
\end{tabular}

Le mécanisme exact de l'hépatotoxicité associée à l'amiodarone n'est pas complètement élucidé, mais le médicament semble interférer avec la ß-oxydation des mitochondries des cellules hépatiques. Cet effet entraine une microstéatose provoquant l'apoptose des hépatocytes ${ }^{4.7}$. Les transaminases hépatiques étant des marqueurs de cytolyse, il est normal de voir leur nombre s'élever dans un tel contexte. On rapporte aussi que l'amiodarone pourrait être associée à une phospholipidose, soit la formation de complexes d'amiodarone et de phospholipides au sein des lysosomes des cellules ${ }^{2,4,7}$. Il n'est pas, pour l'instant, clairement déterminé si la phospholipidose est un marqueur de toxicité ou simplement un signe d'accumulation du médicament dans le tissu hépatique. Les conséquences de la phospholipidose ne sont pas connues. Dans les études cliniques et dans les rapports de cas répertoriés, certains patients ont subi des biopsies hépatiques lorsque leur bilan hépatique était perturbé. Ces biopsies démontrent que les changements histologiques observés lors d'une hépatotoxicité induite par l'amiodarone sont très similaires à ceux observés dans les cas d'hépatite ou de cirrhose alcoolique ${ }^{2,4,7}$.
L'administration intraveineuse d'amiodarone présente une particularité supplémentaire. Certains auteurs croient que le Polysorbate 80, un diluant utilisé dans la composition parentérale d'amiodarone, favorise les réactions d'hépatotoxicitél En effet, l'inhibition de la glycoprotéine-P et du cytochrome P450 3A4 par le Polysorbate 80 permet l'augmentation des concentrations plasmatiques de l'antiarythmique $e^{4,6,8}$.

Dans le cas de cette patiente, certaines données importantes qui aideraient à mieux comprendre la séquence des évènements sont manquantes. En effet, plusieurs jours se sont écoulés sans qu'aucune valeur d'enzymes hépatiques ne soit mesurée, ce qui aurait permis d'avoir un meilleur portrait de leur évolution dans le temps. De plus, d'autres médicaments administrés à la patiente durant son hospitalisation auraient potentiellement pu contribuer à l'atteinte hépatique de la patiente.

Outre l'amiodarone, la pravastatine et le furosémide sont deux molécules pour lesquelles une élévation des transaminases a été rapportée. L'aspirine a aussi été associée à une possible toxicité hépatique, mais l'atteinte hépatocellulaire causée par ce médicament consiste plutôt en une nécrose généralisée des 
hépatocytes, ce qui ne semble pas avoir été le cas ici. D'autres médicaments apparaissant au profil de la patiente, tels la cloxacilline, le métoprolol, l'ésoméprazole, la lévothyroxine et la venlafaxine, peuvent également créer une hépatotoxicité, mais les cas sont beaucoup plus rares et ont été peu rapportés 5 .

Deux constats viennent appuyer la mise en cause de l'amiodarone. Le premier est l'importante augmentation des valeurs des enzymes hépatiques à la suite de l'introduction de l'amiodarone, et le second est une amélioration du bilan hépatique après son retrait. La relation temporelle est donc plus évidente qu'avec les autres médicaments administrés à la patiente. Cette observation corrèle avec les conclusions rapportées dans la littérature scientifique mentionnées plus haut. En utilisant l'algorithme de Naranjo pour établir un degré de corrélation entre l'amiodarone et l'effet indésirable observé, le score obtenu est de 3 . Le lien peut être qualifié de possible ${ }^{11}$. Considérant l'historique d'hospitalisation de la patiente et le lien de causalité dans le temps, nous pensons que l'amiodarone est l'agent responsable de l'hépatotoxicité.

\section{CONCLUSION}

En conclusion, l'amiodarone est associée à de nombreux effets indésirables, dont différents types de toxicité hépatique, allant de l'élévation légère et transitoire des transaminases, tel que ce cas l'a démontré, à la dysfonction hépatique grave. Il convient donc de faire un suivi rigoureux des enzymes hépatiques tant auprès des patients recevant un traitement à long terme à l'amiodarone qu'auprès de ceux traités pendant une courte durée. Il n'y a cependant pas de recommandations claires concernant le suivi à court terme des patients commençant un traitement à l'amiodarone, ni en ce qui a trait à la gestion du médicament dans un contexte d'augmentation des enzymes hépatiques. Le recours au jugement clinique du personnel soignant est nécessaire pour évaluer au cas par cas la conduite à tenir dans de telles situations. À notre avis, si les ressources le permettent, un suivi hebdomadaire ou bimensuel des enzymes hépatiques serait utile durant le premier mois de traitement. Les patients traités à l'amiodarone à long terme devraient subir des tests de fonction hépatique au moins tous les six mois ${ }^{12}$. Si la concentration des transaminases hépatiques dépasse le triple de la valeur normale supérieure, ou si on observe que le taux double chez un patient ayant déjà un niveau de base élevé, il est raisonnable d'envisager une diminution ou, idéalement, le retrait de l'amiodarone. Bien évidemment, il est primordial d'avoir d'abord considéré les risques et les bienfaits associés à l'arrêt du traitement et de s'assurer de bien prendre en charge tous les aspects liés à la pathologie du patient ${ }^{5,10}$.

\section{Références}

1. Santangeli P, Di Biase L, Burkhardt JD, Bai R, Mohanty P, Pump A, et coll. Examining the safety of amiodarone. Expert Opin Drug Saf. 2012;11(2):191-214.

2. Amiodarone hydrochloride. Dans : AHFS Drug Information 2012. Bethesda (MD) : American Society of Health-System Pharmacists; 2012. Publié au : www.ahfsdruginformation.com. Consulté le 15 avril 2012.

3. Giardina EG, Zimetbaum PJ, Link M, Downey BC. Major side effects of amiodarone. Dans : UpToDate 20.3 [base de données en ligne]. Wolters Kluwer; 2012. Publié au : www.uptodate.com. Consulté le 15 avril 2012.

4. Mattar W, Juliar B, Gradus-Pizlo I, Kwo PY. Amiodarone hepatotoxicity in the context of the metabolic syndrome and right-sided heart failure. $J$ Gastrointestin Liver Dis. 2009;18(4):419-23.

5. Amiodarone hydrochloride. Dans : Micromedex health series [base de données en ligne]. Greenwood Village (CO) : Thomson Reuters; 2012. Publié au : www.thomsonhc.com/micromedex2. Consulté le 15 avril 2012.

6. Rao U, Agarwal A. Amiodarone-induced acute hepatotoxicity. Eur J Clin Pharmacol. 2012;68(4):449-50.

7. Sung PS, Yoon SK. Amiodarone hepatotoxicity. Hepatology. 2012; 55(1):325-6

8. Aronson JK, éditeur. Amiodarone. Dans : Meyler's side effets of drugs: the international encyclopedia of adverse drug reactions and interactions. $15^{\circ}$ éd. Oxford (UK) : Elseiver; 2006. pages 148-73.

9. Vorperian VR, Havighurst TC, Miller S, January CT. Adverse effects of low dose amiodarone: a meta-analysis. J Am Coll Cardiol. 1997;30(3):791-8.

10. Cordarone. Dans : e-CPS [en ligne]. Ottawa $(\mathrm{ON})$ : Association des pharmaciens du Canada; 2012. Publié au : www.e-therapeutics.ca/. Consulté le 15 avril 2012

11. Naranjo CA, Busto U, Sellers EM, Sandor P, Ruiz I, Roberts EA, et coll. A method for estimating the probability of adverse drug reactions. Clin Pharmacol Ther. 1981;30(2):239-45.

12. Sanoski CA, Schoen MD, Bauman JL. The arrythmias. Dans : DiPiro JT, Talbert RL, Yee GC, MatzkeGR, Wells BG, Posey LM, rédacteurs. Pharmacotherapy: a pathophysiologic approach. $7^{\circ}$ éd. New York: McGrawHill Companies Inc; 2008. Pages 279-314.

Jessica Ferguson, Pharm. D., M. Sc., était résidente au Centre hospitalier de I'Université de Montréal au moment de la rédaction de cet article et est maintenant pharmacienne à ce même centre à Montréal, Québec.

Anne-Isabelle Dubé, Pharm. D., M. Sc., était résidente au Centre hospitalier de I'Université de Montréal au moment de la rédaction de cet article et est maintenant pharmacienne au Centre universitaire de santé McGill à Montréal, Québec.

Catherine Ouellet, B. Pharm. M. Sc., est pharmacienne au Centre hospitalier de I'Université de Montréal, Montréal, Québec.

Divulgation des intérêts concurrents: Aucun déclarés.

Adresse de correspondance :

Jessica Ferguson

Pharmacie

Centre hospitalier de I'Université de Montréal

6757, avenue de Gaspé

Montréal QC H2S 2Y6

Courriel : jessica.ferguson@umontreal.ca 